\title{
Distance Measurement System using A Stereo Camera and Radial Pattern Target for Automatic Berthing Control
}

\author{
Yoshiaki Mizuchi ${ }^{\star \dagger}$ Tadashi Ogura*, Yoshinobu Hagiwara**, \\ Akimasa Suzuki***, Youngbok Kim ${ }^{\star * * *}$ and Yongwoon Choi ${ }^{*}$
}

(received 22 September 2013, revised 05 October 2013, accepted 06 October 2013)

\begin{abstract}
In this paper, we propose a distance measurement system for automatic berthing control using a stereo camera mounted on a rotation control device, and a radial pattern target. Automatically controlling the position and attitude of a ship aims to prevent maritime accidents due to human error. Our goal is to measure the relative distance between a ship and an onshore or offshore target for berthing. Therefore, the distance should be continuously measured while tracking a fixed point on a target. To this end, we developed a stereo camerabased distance measurement system that satisfied these requirements. This paper describes the structure and principle of the measurement system. We validate the distance error for target incline due to the relative position and attitude between a camera and a target in miniature scale. In addition, the findings of an experiment in an outdoor environment demonstrate that the proposed measurement system has accuracy within $1 \mathrm{~m}$ at a range of $20-100 \mathrm{~m}$ which is the acceptable accuracy for automatic berthing.
\end{abstract}

Key Words : Automatic Berthing Control, Distance Measurement, Stereo Camera, Template Matching.

\section{Introduction}

To load or unload cargoes from wharfs or floating production, storage and offloading (FPSO), ocean vessels are berthed alongside. During the maneuvering and berthing process, the position and attitude of ocean vessels should be continuously

$* \dagger$ Yoshiaki Mizuchi(corresponding author), Tadashi Ogura, Yong-Woon Choi: Department of Information System Science, Soka University, Japan.

E-mail: e12d5202@soka.ac.jp, Tel : +81-42-691-8197.

**Yoshinobu Hagiwara: National Institute of Informatics, Japan.

***Akimasa Suzuki: Department of Software and Information Science, Iwate Prefectural University, Japan.

**** Youngbok Kim: Department of System Mechanical Engineering, Pukyong National University. adjusted for an extended period. If operators are required to concentrate on the maneuvering position and attitude of ocean vessels continuously, the risk of maritime accidents induced by psychological and physical fatigue is increased ${ }^{1)}$. Automatic berthing control is an approach to relieving operator fatigue. This is achieved by continuously measuring the relative distance from a ship to a berthing destination. Furthermore, the ideal measurement accuracy is within $1 \mathrm{~m}$ for the measurement range $20-100 \mathrm{~m}$ in outdoor environments.

Although the global positioning system (GPS) is widely used in ship navigation systems ${ }^{2)}$, the positional accuracy provided by GPS is generally 
insufficient for berthing. As a precise and real-time GPS-based approach, Ueno has proposed a positioning system ${ }^{3)}$ for automatic ship berthing using real-time kinematic (RTK) GPS techniques. However, this system is not suitable for remote offshore facilities, since it requires GPS receivers that are fixed to the ground for accurate calibration. On the other hand, laser sensors are widely used as relative distance measurement devices for berthing aid systems ${ }^{4)}$. Laser distance measurement sensors, generally placed on a wharf, have measurement accuracy within $1 \mathrm{~m}$. However, when a laser sensor is applied to automatic berthing, it may fail during continuous distance measurements because it is difficult to irradiate the same target point continuously.

In the present study, we propose a distance measurement system using a stereo camera mounted on a rotation control device and a radial pattern target placed on facilities or FPSO vessels. This would enable relative distance measurements while tracking a fixed point on the target. Through automatic and continuous measurement of the distance from a ship to the target, the position and attitude of the ship can be maneuvered.

In this paper, we describe the structure and measurement principle of the proposed system. To validate the robustness of the measurement system against target deformations due to the relative attitude between the camera and target, we experimented in a miniature and indoor environment. In addition, experimental results in an outdoor environment demonstrate that the proposed system has accuracy within $1 \mathrm{~m}$ for a measurement range of 20-100 m.

\section{Distance Measurement System}

\subsection{System Overview}

An overview of the distance measurement system is shown in Fig. 1. This measurement system consists of a stereo camera mounted on a rotation control device, and a specific target. By detecting the same point on a target in each camera image, the distance to the target can be measured by employing the triangulation method. The rotation control device rotates the cameras along its pan and tilt axes to track the target. The target has a type of radial pattern that can be robustly detected against scale transition, which could occur due to the relative distance to the target. To address this issue, our measurement system has the capability of measuring the relative distance between the stereo camera and the target while tracking a fixed point.

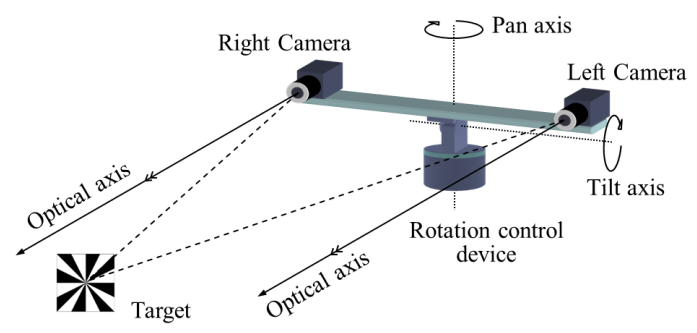

Fig. 1 Overview of distance measurement system using a stereo camera mounted on a rotation control device.

Fig. 2 shows a flowchart of this distance measurement system. The target in each input image of the stereo camera is detected by template matching. In this process, the system selects the most similar template image from a database of pre-recorded deformed target images. This is to accurately detect the position of the deformed target depending on the relative position between each camera and the target. As shown in Fig. 1, the target position in each camera image is related to finding the angle relative to the optical axis of each camera. The distance to the target is calculated based on the obtained angles and the 
baseline length between the cameras. In addition, the rotation control device turns the stereo camera toward the target. Through continuous tracking of the stereo camera toward the target, the system can track the target and measure the distance to the target simultaneously.

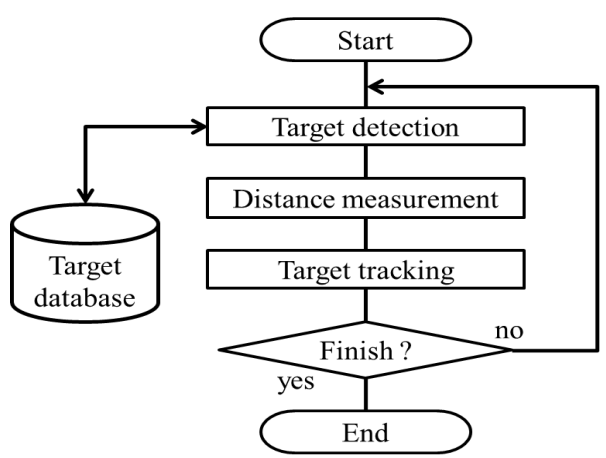

Fig. 2 Flowchart of distance measurement using our system.

\subsection{Target Detection}

The target position in an input image is detected by template matching using vector code correlation $(\mathrm{VCC})^{5 \sim 7)}$. VCC is a simple calculation method suitable for tracking a target. Furthermore, it has been demonstrated in previous studies that VCC is applicable to target detection in outdoor lighting conditions $^{5 \sim 6}$ and bad weather ${ }^{7)}$. Fig. 3 shows the target detection procedure using the VCC method. The dashed square in the input image shows a detection window to find the given target image. The solid square in the detection window shows a $3 \times 3$ pixel area to generate a four-bit code for the gradient. As shown in the upper half of Fig. 3, the horizontal and vertical gradients for each pixel area in the detection window, denoted as $G_{h}$ and $G_{v}$ respectively, are obtained through horizontal and vertical filters. $G_{h}$ and $G_{v}$ are encoded into three types of gradient vector codes, that is 01 (positive), 00 (neutral), and 10 (negative). The generated vector codes of an input image and a target image are simply compared with each other by using bitwise exclusive-OR. For example, in Fig. 3, the number of ' 1 's is obtained as dissimilarity in a pixel area. Thus, the area with the lowest summation of dissimilarity is detected as the target area.

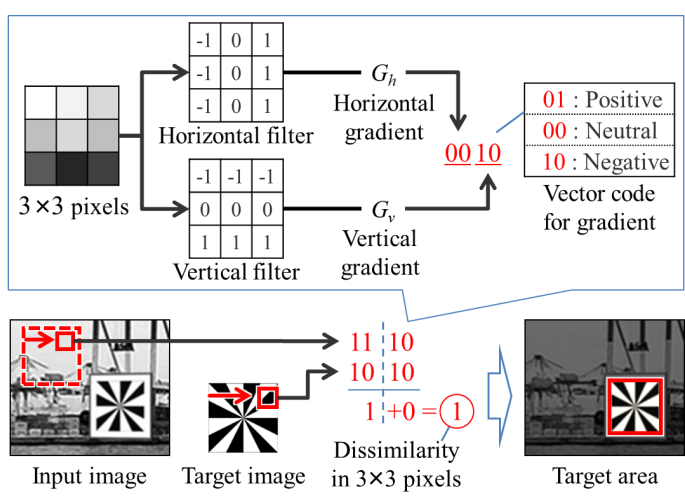

Fig. 3 Procedure of target detection using VCC.

When a target is not captured from the front but from an oblique angle, the target in an input image would be deformed. For example, a target is deformed based on the relative position and attitude of a ship, or the height difference between the cameras and a target position. If the captured shape of the target is excessively deformed, the detecting location would be misaligned from the actual target location. Accordingly, if the measurement was conducted using the misaligned location, the measurement error would be increase. For this reason, the closest template image match in the deformation database, as shown in Fig. 4, is selected and applied for detection. This database consists of 81 pre-recorded deformed target images that are rotated about the vertical and horizontal axes from -40 to $+40[\mathrm{deg}]$ at 10 [deg] intervals relative to the front image. In addition, each central point of the target pattern is adjusted to coincide with each other. Selection of the best correlated target, allows a precise measurement to 
be achieved.

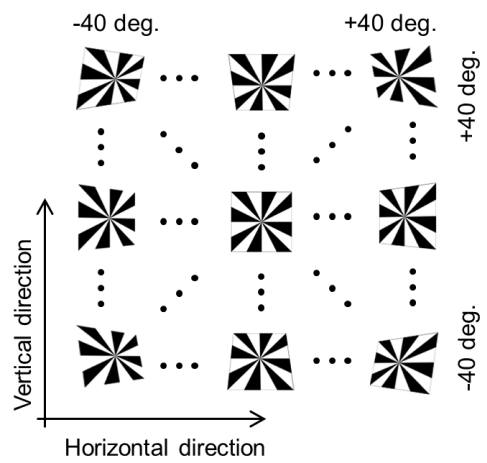

Fig. 4 Pre-recorded images as a database for target deformation.

\subsection{Distance Measurement}

Fig. 5 shows the parameters of angle calculation for distance measurement using a target location in an input image. As shown in Fig. 5, the target location in the input image is given as coordinates $\left(u_{t}, v_{t}\right)$ by target detection. The target location denotes the pixel location relative to the intersection of the image plane and the optical axis of the camera. Angle $\theta$ in Fig. 5 between the optical axis and the target is calculated by the following equation:

$$
\theta=\tan ^{-1} \frac{u_{t} s_{u}}{f}
$$

where $s_{u}$ denotes the horizontal pixel size and $f$ is

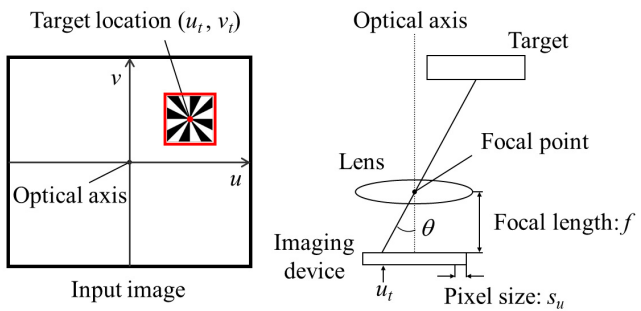

Fig. 5 Parameters of angle calculation for distance measurement. the physical focal length of the lens. We assume that $s_{u}$ and $f$ are known values in the measurement system.

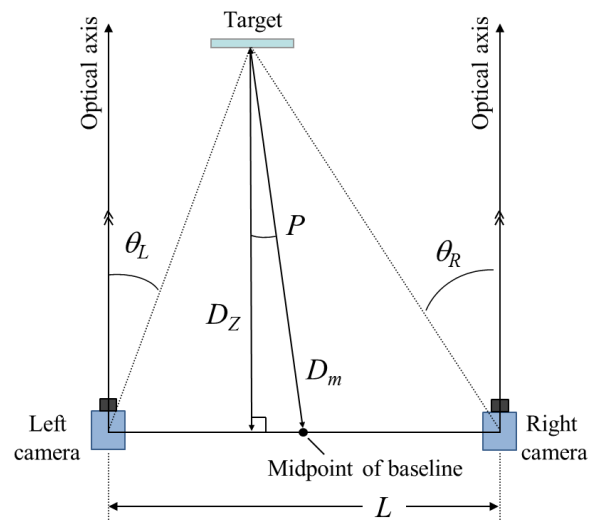

Fig. 6 Overview of distance measurement using two target angles.

Fig. 6 shows an overview of the distance measurement using two target angles obtained from each camera. Here, $\theta_{L}$ and $\theta_{R}$ denote the obtained angles of the left and right cameras from equation (1), respectively. The distance $D_{Z}$ between the baseline and the target is computed from $\theta_{L}$ and $\theta_{R}$ :

$$
D_{z}=\frac{L}{\tan \theta_{L}-\tan \theta_{R}}
$$

where $L$ denotes the baseline length. Since the stereo camera is rotated for tracking, the distance relative to the pan axis of rotation control device should be measured. In our system, the axis passes through the midpoint of the baseline. Thus, the distance $D_{m}$ between the midpoint and a target is obtained, as:

$$
D_{m}=\sqrt{\frac{1}{2} D_{z}\left(\tan ^{2} \theta_{L}+\tan ^{2} \theta_{R}+2\right)-\frac{L^{2}}{4}}
$$


We assumed the distance $D_{m}$ to be the distance between the measurement system and the target point.

\subsection{Target Tracking}

To continuously measure the distance, the stereo camera is rotated by the rotation control device such that the target is centrally located in the input image. Pan angle $P$ of the rotation control device is derived from the previously mentioned $D_{m}$ and $D_{z}$ as:

$$
P=\cos ^{-1}\left(\frac{D_{z}}{D_{m}}\right)
$$

Tilt angle $T$ is derived from the equation (1):

$$
T=\tan ^{-1} \frac{v_{t} s_{v}}{f}
$$

where each variable is shown in Fig. 5. Through tracking the center point of a target using $P$ and $T$, the measurement system can continuously measure the distance.

\section{EXPERIMENTS}

We conducted two experiments to determine if the proposed system has the robustness against the target deformation and satisfies the required measurement accuracy in an actual range. These experiments were performed with each prototype system. The results show the potential of the proposed system for distance measurement for automatic berthing control.

\subsection{Target Deformation Experiment}

Fig. 7 shows an experimental prototype system in miniature scale for confirming the robustness of the distance measurement system under target deformation condition. As shown in Table 1, the baseline of the stereo camera is $178 \mathrm{~mm}$, and the focal length and the resolution of each camera are $16 \mathrm{~mm}$ and $640 \times 480$, respectively. The horizontal pixel size is $7.5 \mu \mathrm{m}$. The target is placed 3000 $\mathrm{mm}$ from the stereo camera at a 30 -degree incline.

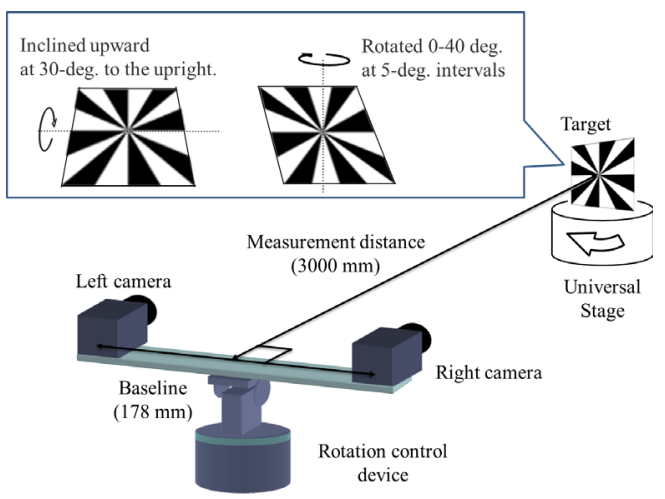

Fig. 7 Experimental prototype system for target deformation with miniature scale.

Table 1 Experimental setup of prototype system for miniature scale.

\begin{tabular}{|c|c|}
\hline Baseline length & $178 \mathrm{~mm}$ \\
\hline Focal length & $16 \mathrm{~mm}$ \\
\hline Image resolution & $640 \times 480$ \\
\hline Pixel size & $7.5 \mu \mathrm{m}$ \\
\hline
\end{tabular}

The distance between the camera and the target was measured while the universal stage was horizontally rotated at 5-degree intervals between 0-40 degrees. The target deformation database, comprising the 81 pre-recorded deformed images, as described in the previous section, was used in the distance measurement procedure.

The results of the measurement accuracy experiment based on the target deformation are shown in Fig. 8. Fig. 8 shows measurement errors and standard deviations from 100 successive image frames. In each graph, the horizontal axis corresponds to the rotation angle of the target. In Fig. 8 (a), the average distance errors are plotted against error range. The average errors are 
uncorrelated with angles of target. Similarly, there is no correlation between the standard deviations and the target angles as shown Fig. 8 (b). Therefore, this demonstrates that the system is able to measure the distance regardless of the target deformation.

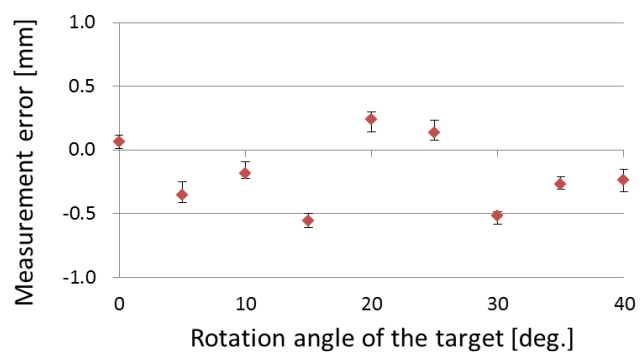

(a) Measurement errors

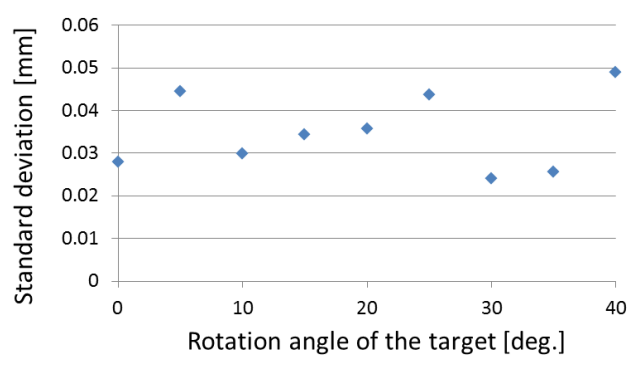

(b) Standard deviations

Fig. 8 Results of distance measurement.

\subsection{Distance Measurement Experiment}

To determine if the proposed system satisfies the required measurement accuracy in an actual range and in an outdoor environment, the distance was measured using the other prototype system under the scenario shown in Fig. 9. As shown in Table 2, the focal length of each camera and the base line are $50 \mathrm{~mm}$ and $1540 \mathrm{~mm}$, respectively. The other parameters are identical to the previous experiment. In this experiment, distance is measured in the range $20-100 \mathrm{~m}$ at $1 \mathrm{~m}$ intervals.

Fig. 10 shows the average distance errors and error ranges in 100 successive image frames. The vertical and horizontal axes correspond to the

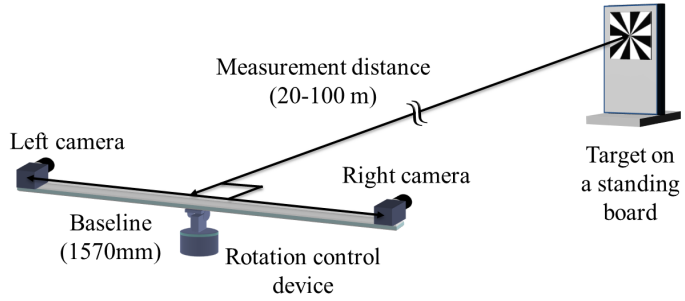

Fig. 9 Experimental prototype system for distance measurement in actual range.

Table 2 Experimental setup of prototype system for distance measurement in actual range.

\begin{tabular}{|c|c|}
\hline Focal length & $50 \mathrm{~mm}$ \\
\hline Baseline length & $1540 \mathrm{~mm}$ \\
\hline Image resolution & $640 \times 480$ \\
\hline Pixel size & $7.5 \mu \mathrm{m}$ \\
\hline
\end{tabular}

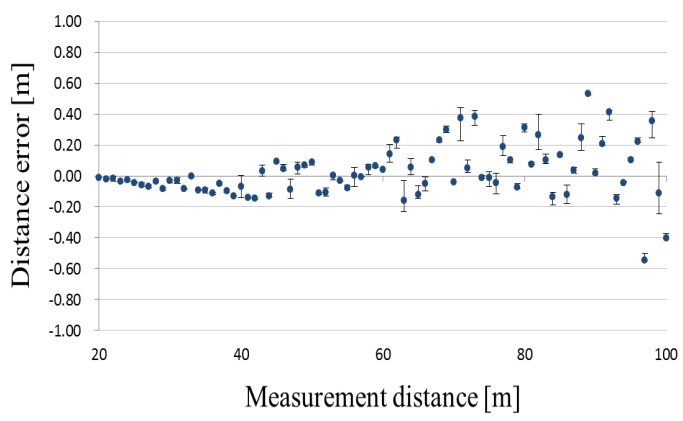

Fig. 10 Result of distance measurement for actual range.

distance error and the actual distance to the target, respectively. The actual distance from the midpoint of the baseline to the target was measured by a laser distance meter which has an accuracy of $1.5 \mathrm{~mm}$. The maximum distance measurement error is 0.551 $\mathrm{m}$. Hence, the proposed system has accuracy within $1 \mathrm{~m}$ for the measurement range 20-100 m which satisfies the required accuracy. In addition, each error is less than 0.62 percent relative to the actual distance. It should be noted that measurement errors are reduced closer to the 
target. This is a suitable feature for berthing, because the risk of accidents would increase as a ship approaches a facility. In summary, these experimental results demonstrate that the proposed measurement system is an effective solution for automatic berthing.

\section{CONCLUSION}

In this paper, we proposed a distance measurement system using a stereo camera in the field of ship automation and control. We also described the structure and measurement principle of the system. Based on the findings of the scale experiment, we verified the system's ability to measure the distance regardless of the target deformation. This result demonstrates its robustness against changes in the relative attitude and position between a ship and a berthing destination. The other experiment demonstrates that the proposed system has accuracy within $1 \mathrm{~m}$ for the measurement range 20-100 $\mathrm{m}$. Therefore, the proposed distance measurement system is an effective solution for automatic berthing control.

In future work, we would like to evaluate the effectiveness of our system in detail, for example, measurement accuracy under adverse weather conditions. To validate the ship automation and control system presented in this paper, we would also like to investigate attitude measurement techniques of a ship using our automated system.

\section{Acknowledgement}

This work was supported by the Basic Science Research Program funded by the Ministry of Education Science and Technology (2012R1A1A22039012). Also, this work was a part of the project titled: "The Development of Mooring Positioning Control System for Offshore
Accommodation Barge" funded by the Ministry of Oceans and Fisheries.

\section{References}

1. C. C. Baker, and D. B. MaCafferty, 2005, "Accident database review of human-element concerns: what do the results mean for classification?," Proc. of International conference Human Factor in Ship Design, Safety and Operation, Royal Institution of Naval Architects.

2. Marine GPS Navigator GP150, http://www.furuno.com/en/business_product/pdf/ma rine/gp150.pdf, Furuno Electric Co., Ltd.

3. M. Ueno, 2000, "A GPS-based system for precise shipping guidance and control”, Journal of Marine Science and Technology, Vol. 5, No. 1, pp. 9-15.

4. SRD-303i Ship's Docking Aid System, http://www.koden-electronics.co.jp/eng/industrial/sr d303i-e.html, Koden Electronics Co., Ltd.

5. H. Kawai, Y. B. Kim, and Y. Choi, 2009, "Anti-sway system with image sensor for container cranes", Journal of mechanical science and technology, Vol. 10, No. 23, pp. 2757-2765.

6. H. Kawai, Y. Choi, Y. B. Kim, and Y. Kubota, 2009, "Measurement system design for sway motion based on image sensor", Proc. of IEEE International Conference on Networking, Sensing and Control, pp. 185-188.

7. H. Kawai, Y. B. Kim, and Y. Choi, 2012, "Measurement of a Container Crane Spreader Under Bad Weather Conditions by Image Restoration", IEEE Trans. on Instrumentation and Measurement, Vol. 1, No. 61, pp. 35-42. 